\title{
Distinction of hepatocellular adenoma from hepatocellular carcinoma with and without cirrhosis using E-cadherin and matrix metalloproteinase immunohistochemistry
}

\author{
Maria S Tretiakova ${ }^{1}$, John Hart ${ }^{1}$, Meer Taher Shabani-Rad ${ }^{2}$, Jianguo Zhang ${ }^{3}$ and \\ Zu-hua $\mathrm{Gao}^{2}$ \\ ${ }^{1}$ Department of Pathology, University of Chicago Hospitals, Chicago, IL, USA; ${ }^{2}$ Department of Pathology and \\ Laboratory Medicine, University of Calgary and Calgary Laboratory Services, Calgary, AB, Canada and \\ ${ }^{3}$ Department of Medicine, University of Calgary, Calgary, AB, Canada
}

\begin{abstract}
We studied the tumor cell expression patterns of E-cadherin and matrix metalloproteinase-1, $-2,-7$, and -9 in a tissue microarray composed of 20 normal livers, 10 hepatocellular adenomas, 43 hepatocellular carcinomas with cirrhosis and 33 hepatocellular carcinomas without cirrhosis. Hepatocellular adenoma was characterized by the complete absence of matrix metalloproteinase-7 expression; hepatocellular carcinoma with cirrhosis was characterized by a significantly low expression of E-cadherin; and hepatocellular carcinoma without cirrhosis was characterized by low matrix metalloproteinase-9 expression. The staining intensity score of E-cadherin $=3$, matrix a metalloproteinase- $7<1$, and matrix metalloproteinase- $9 \geq 2$ can be used as the diagnostic criteria for hepatocellular adenoma and for distinguishing hepatocellular adenoma from normal, hepatocellular carcinoma with cirrhosis, and hepatocellular carcinoma without cirrhosis. E-cadherin $<2$ and matrix metalloproteinase-9 $<2$ can be used for distinguishing both hepatocellular carcinoma with cirrhosis and hepatocellular carcinoma without cirrhosis from normal. Although statistically not significant, hepatocellular carcinoma without cirrhosis showed a higher E-cadherin expression and a lower matrix metalloproteinase-9 expression than hepatocellular carcinoma with cirrhosis, which could be partially responsible for the less aggressive behavior found in hepatocellular carcinoma without cirrhosis when compared with hepatocellular carcinoma with cirrhosis. These results, if confirmed in a further study of small biopsy specimens and of histologically ambiguous cases, could lead to the application of these markers in the diagnosis and differential diagnosis of hepatocellular neoplasms in our surgical pathology practice.

Modern Pathology (2009) 22, 1113-1120; doi:10.1038/modpathol.2009.75; published online 22 May 2009
\end{abstract}

Keywords: hepatocellular carcinoma; adenoma; cirrhosis; metalloproteinase; E-cadherin

Primary hepatocellular neoplasm can be broadly classified into malignant and benign categories. Hepatocellular carcinoma is the sixth most common cancer worldwide in terms of numbers of cases (626 000/year), but because of its very poor prognosis, it is the third most common cause of death from cancer (598 000/year). ${ }^{1}$ The major risk factor for the development of hepatocellular carcinoma is liver cirrhosis associated with chronic hepatitis B

Correspondence: Dr Zu-hua Gao, MD, PhD, Department of Pathology and Laboratory Medicine, Foothills Medical Center, The University of Calgary, Room C1150E, 1403-29 Street NW, Calgary, Alberta, Canada T2N 2T9, USA.

E-mail: zu-hua.gao@cls.ab.ca

Received 14 February 2009; revised 20 April 2009; accepted 21 April 2009; published online 22 May 2009 and hepatitis C infection, alcohol, aflatoxin B exposure, and various metabolic disorders. ${ }^{2-5}$ In approximately $15-20 \%$ of patients, hepatocellular carcinoma arises from a non-cirrhotic liver. ${ }^{6}$ On the bases of etiology and the frequency of occurrence, hepatocellular carcinoma without cirrhosis can be divided into four groups: (1) rarely occurring in patients with viral hepatitis and alcohol abuse; (2) frequently occurring in patients with $\alpha-1$ antitrypsin deficiency, hemochromatosis, and non-alcoholic fatty liver disease; (3) consistently occurring in patients with glycogen storage disease type 1 and consumption of oral contraceptives/anabolic steroids; and (4) unidentifiable underlying etiology as is reported in the majority of cases. ${ }^{7}$ Hepatocellular adenoma is an uncommon benign liver cell neoplasm with an estimated prevalence of $1-3$ per 
100000 in young women with long-standing contraceptive use. Other etiological factors for the development of hepatocellular adenoma include glycogen storage disease type 1, therapy with estrogen and gestagen as well as anabolic corticosteroids. ${ }^{8,9}$ Survival advantages of patients with hepatocellular carcinoma without cirrhosis over those with cirrhosis have been well documented. ${ }^{6,10,11}$ This survival difference is believed to be due to the poor liver functional reserve in a cirrhotic liver and a tendency toward development of a new primary tumor after surgery in this setting. ${ }^{12,13}$ The clinical behavior of hepatocellular adenoma includes regression after cessation of oral contraceptives, remaining stable, an increase in size, bleeding, or rarely undergoing a malignant transformation. ${ }^{14}$ Patients with appropriately resected hepatocellular adenomas are usually inconsequential. Therefore, it is of paramount importance to clearly differentiate between these three types of primary liver tumor because of the significant differences in their clinical behaviors and therapeutic approaches. However, this task can be very difficult to accomplish in small biopsy specimens.

Invasion and metastasis are the biological hallmarks of malignancy and the major causes of cancerrelated morbidity and mortality. Breaching through the basement membrane and interstitial extracellular matrix is the key process for tumor invasion, which requires the action of a series of proteolytic enzymes named matrix metalloproteinases (MMPs). ${ }^{15-18}$ Dispersion of tumor cells from the primary tumor is one of the key events for tumor metastasis, which relies on the loss of homotypic cell-cell adhesion, a process mediated by the E-cadherin/catenin complex. ${ }^{19}$ We have reported earlier the association of E-cadherin, MMPs, and TIMPs with the progression and metastasis of hepatocellular carcinoma. ${ }^{20}$ In this study, we examined the tumor cell expression patterns of E-cadherin, MMP-1, -2, -7, and -9 in a tissue microarray composed of normal liver, hepatocellular adenoma, hepatocellular carcinoma with cirrhosis, and hepatocellular carcinoma without cirrhosis. The aim of this study was to clarify the differences of the expression of these biological markers in histologically unequivocal benign and malignant primary hepatocellular neoplasms, and to identify biological markers that might be helpful in the differential diagnosis of small biopsy specimens and of histologically ambiguous cases.

\section{Materials and methods}

\section{Tissue Samples}

With the approval of the institutional review board, we retrieved paraffin blocks of the following surgically resected tissue samples from the Department of Pathology, University of Calgary and the Department of Surgical Pathology, University of Chicago Hospitals: 20 normal livers (15 men and 5 women; age range 29-100 years), 10 hepatocellular adenomas (all women; age range $20-50$ years, mean 30.5 years), 43 hepatocellular carcinomas with cirrhosis (23 men and 20 women; age range $44-82$ years), and 33 hepatocellular carcinomas without cirrhosis (22 men and 11 women; age range 25-85 years). Routine hematoxylin-eosin-stained tissue sections were reviewed by two pathologists $(\mathrm{JH}$ and $\mathrm{ZG}$ ) and the tumors were graded using the World health Organization grading system. ${ }^{21}$ Hepatocellular carcinoma without cirrhosis was defined by the lack of bridging fibrosis in the background non-neoplastic liver tissue. Three representative areas from each tumor were selected for the construction of the tissue microarray blocks using 1.0-mm punchers on the manual tissue arrayer MTA-1 (Beecher Instruments, Sun Prairie, WI, USA).

\section{Immunohistochemical Analysis}

Immunohistochemical staining was performed on $4-\mu \mathrm{m}$ sections obtained from formalin-fixed, paraffin-embedded tissue microarray blocks. After deparaffinization and rehydration, tissue sections were incubated with monoclonal antibodies against E-cadherin, MMP-1, -2, -7, and -9 (Table 1). A subsequent reaction was performed with biotinfree horseradish peroxidase-labeled polymer from the EnVision plus detection system (DakoCytomation, Carpinteria, CA, USA). A positive reaction was visualized using diaminobenzidine solution followed by counterstaining with hematoxylin. Positive controls were selected according to the manufacturers' recommendations: squamous epithelium for E-cadherin; placenta, bladder, breast, and ovarian carcinomas for MMP-1, -2 , and -7 ; and macrophages in tonsil tissue for MMP-9. Negative controls were prepared using non-immune mouse or rabbit IgGs.

Table 1 List of antibodies against matrix metalloproteinases and E-cadherin

\begin{tabular}{lllllll}
\hline & MMP-1 & MMP-2 & MMP-3 & MMP-7 & MMP-9 & E-cadherin \\
\hline Isotype & Poly & IgG1 & IgG2b & IgG2b & IgG1 & IgG2b \\
Clone & - & $42-5 \mathrm{D} 11$ & SL-1 & ID2 & 23C & Mouse \\
Species & Rabbit & Mouse & Mouse & Mouse & Mouse \\
Dilution & $1: 750$ & $1: 100$ & $1: 20$ & $1: 25$ & $1: 30$ & $1: 50$ \\
Source & Neomarkers & Oncogene Research Products & Neomarkers & Neomarkers & Novacastra & Novacastra
\end{tabular}


The intensity of membranous staining for E-cadherin and cytoplasmic staining for MMPs was graded blindly by two pathologists (ZG and MT) independently at different times and scored using a conventional four-point scoring system $(0=$ no staining, $1=$ weak staining, $2=$ moderate staining, and $3=$ strong staining). The score of each case was calculated as average intensity score of three separate cores $\times$ estimated percentage of positive cells with increments of $25,50,75$, and $100 \%$. Discrepancies in grading were resolved by simultaneous grading at a multihead microscope in the presence of a third pathologist $(\mathrm{JH})$. The grading results were further verified by the automated Chromavision Cellular Imaging System (Clarient Inc., San Juan Capistrano, CA, USA).

\section{Statistical Analysis}

The data were compiled in a Microsoft Excel spreadsheet and analyzed using STATA 9.0 software (StataCorp., College Station, TX, USA). A nonparametric multiple analysis of variance (MANOVA) was used to see the effect of a categorical variable (normal liver, adenoma, hepatocellular carcinoma with cirrhosis, and hepatocellular carcinoma without cirrhosis) on multiple dependent variables (MMP-1, -2, -7, etc). The result shows that the effect can be regarded as significant $(P<0.0001)$. Subsequently, a series of Kruskal-Wallis tests on each dependent variable were used to determine which tissue types differed significantly from others. After setting the diagnostic threshold for each type of tumor, the sensitivity, specificity, and receiver operating characteristic (ROC) of each selected marker, individually or in combination, were calculated using the following standard formula: ${ }^{22,23}$

Sensitivity $=$ the marker score fell within the diagnostic threshold for a particular type of tumor/ case with this type of tumor.

Specificity $=$ the marker score fell outside the diagnostic threshold for a particular type of tumor/ case without this type of tumor.

Receiver operating characteristic $=$ Sensitivity/ 1-Specificity.

\section{Results}

\section{Comparison of Protein Marker Expression Among Different Types of Tissues}

The results are illustrated in Figures 1 and 2, and Table 2.

Compared with normal liver, hepatocellular carcinoma with cirrhosis showed a significantly lower expression of E-cadherin; hepatocellular carcinoma without cirrhosis showed a significantly lower expression of MMP-9; and hepatocellular adenoma showed complete absence of MMP-7 expression.

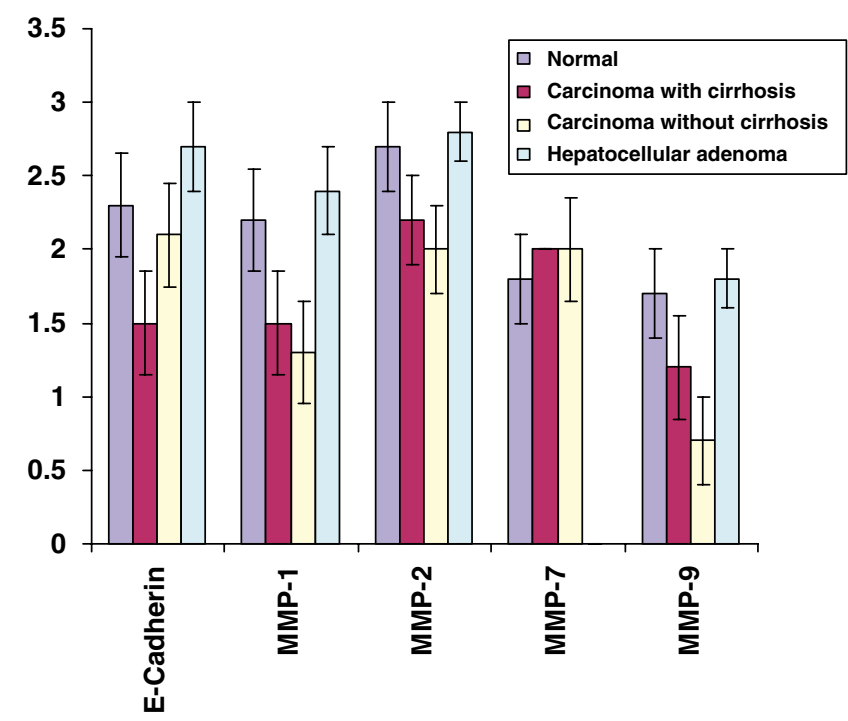

Figure 1 Expression of E-cadherin and matrix metalloproteinases in different liver tissues.

Compared with hepatocellular adenoma, hepatocellular carcinoma with cirrhosis showed a significantly higher expression of MMP-7 and a significantly lower expression of E-cadherin; hepatocellular carcinoma without cirrhosis showed a significantly higher expression of MMP-7 and a significantly lower expression of MMP-9.

There was no significant difference in the expression of MMPs and E-cadherin between hepatocellular carcinoma with cirrhosis and hepatocellular carcinoma without cirrhosis.

\section{Value of Selected Markers in the Diagnosis and Differential Diagnosis}

E-cadherin $=3$, MMP-7 $<1$, and MMP-9 $\geq 2$ were used as the diagnostic criteria for hepatocellular adenoma and for distinguishing hepatocellular adenoma from normal, hepatocellular carcinoma with cirrhosis and hepatocellular carcinoma without cirrhosis. As shown in Table 3, MMP-7 alone is the most sensitive, whereas MMP-7 + MMP-9 is the most specific marker to achieve these objectives.

E-cadherin $<2$ and MMP-9 $<2$ were used for distinguishing hepatocellular carcinoma with cirrhosis and hepatocellular carcinoma without cirrhosis from normal. As shown in Table 3, MMP-9 alone has the highest sensitivity, especially for differentiating hepatocellular carcinoma without cirrhosis from normal. The combination of E-cadherin and MMP-9 can differentiate both hepatocellular carcinoma with cirrhosis and hepatocellular carcinoma without cirrhosis from normal with high specificity, but the sensitivity was very low.

As highlighted in Table 2, E-cadherin and MMPs cannot be used to distinguish hepatocellular 

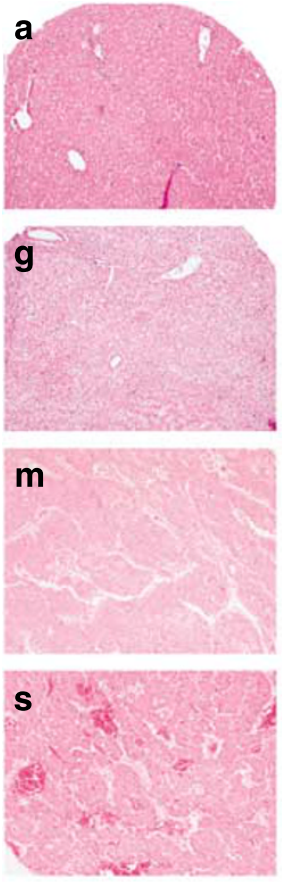
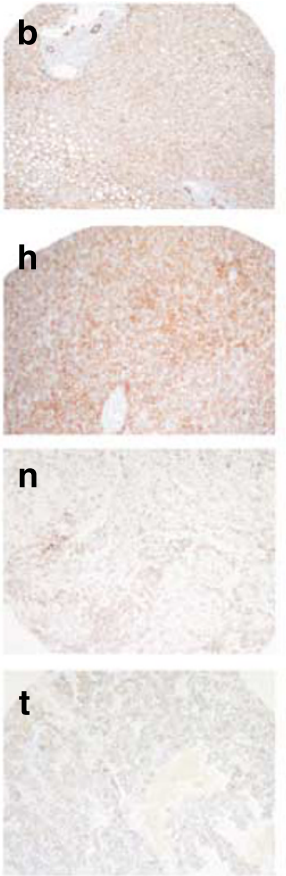
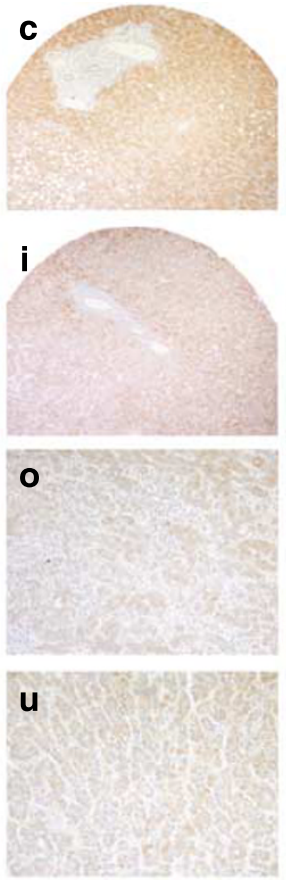
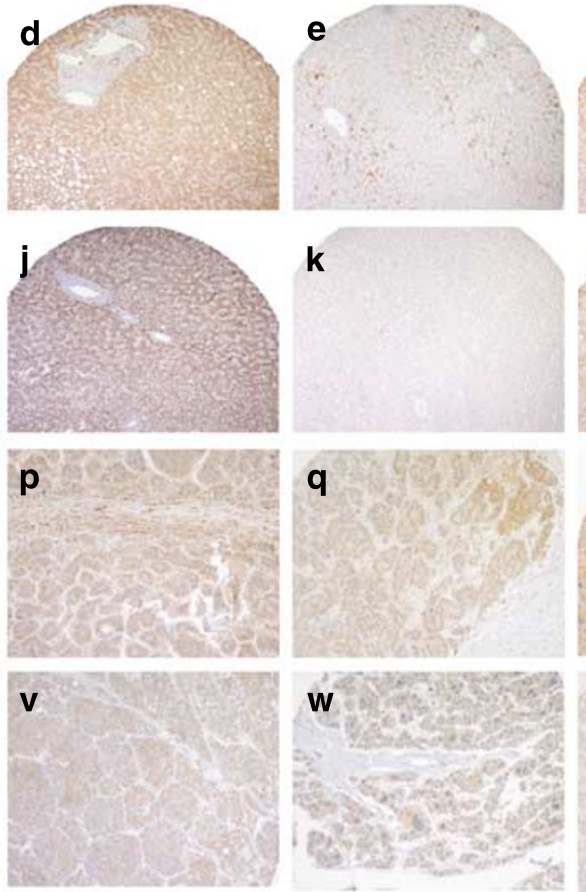
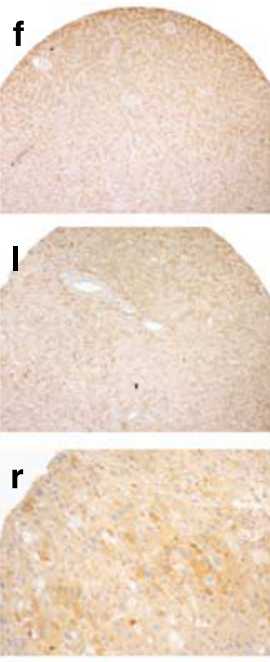

$\mathbf{x}$

$\mathbf{X}$

Figure 2 Representative histology $(\mathrm{H} \& \mathrm{E}, \times 200)$ and expression patterns of E-cadherin and matrix metalloproteinases (immunoperoxidase, $\times 200$ ) in different liver tissues. (a) Histology of normal liver; (b) E-cadherin expression in normal liver; (c) MMP-1 expression in normal liver; (d) MMP-2 expression in normal liver; (e) MMP-7 expression in normal liver; (f) MMP-9 expression in normal liver; (g) histology of adenoma; (h) E-cadherin expression in adenoma; (i) MMP-1 expression in adenoma; (j) MMP-2 expression in adenoma; (k) MMP-7 expression in adenoma; (l) MMP-9 expression in adenoma; (m) histology of hepatocellular carcinoma with cirrhosis; (n) Ecadherin expression in hepatocellular carcinoma with cirrhosis; (o) MMP-1 expression in hepatocellular carcinoma with cirrhosis; (p) MMP-2 expression in hepatocellular carcinoma with cirrhosis; (q) MMP-7 expression in hepatocellular carcinoma with cirrhosis; (r) MMP-9 expression in hepatocellular carcinoma with cirrhosis; (s) histology of hepatocellular carcinoma without cirrhosis; (t) Ecadherin expression in hepatocellular carcinoma without cirrhosis; (u) MMP-1 expression in hepatocellular carcinoma without cirrhosis; (v) MMP-2 expression in hepatocellular carcinoma without cirrhosis; (w) MMP-7 expression in hepatocellular carcinoma without cirrhosis; $(\times)$ MMP-9 expression in hepatocellular carcinoma without cirrhosis.

Table 2 Comparison of tissue expression of matrix metalloproteinases and E-cadherin ( $P$-value by Kruskal-Wallis test)

\begin{tabular}{|c|c|c|c|c|c|}
\hline & E-cadherin & $M M P-1$ & $M M P-2$ & $M M P-7$ & $M M P-9$ \\
\hline Normal vs hepatocellular carcinoma with cirrhosis & $<0.05$ & NS & NS & NS & NS \\
\hline Normal vs hepatocellular carcinoma without cirrhosis & NS & NS & NS & NS & $<0.01$ \\
\hline Normal vs hepatocellular adenoma & NS & NS & NS & $<0.01$ & NS \\
\hline Hepatocellular carcinoma with cirrhosis vs hepatocellular adenoma & $<0.01$ & NS & NS & $<0.01$ & NS \\
\hline Hepatocellular carcinoma without cirrhosis vs hepatocellular adenoma & NS & NS & NS & $<0.05$ & $<0.01$ \\
\hline $\begin{array}{l}\text { Hepatocellular carcinoma with cirrhosis vs hepatocellular } \\
\text { carcinoma without cirrhosis }\end{array}$ & NS & NS & NS & NS & NS \\
\hline
\end{tabular}

MMP, matrix metalloproteinase.

carcinoma with cirrhosis from hepatocellular carcinoma without cirrhosis.

\section{Discussion}

E-cadherin, a transmembrane glycoprotein, mediates $\mathrm{Ca}^{2+}$-dependent cell-cell adhesion through its intracytoplasmic interaction with $\beta$ - and $\alpha$-catenin. $\alpha$-Catenin connects the cadherin-catenin complex to actin filament networks, leading to increased adhesive strength. Changes in adhesion complexes lead to alterations of cell polarity, proliferation, mobility, and differentiation. ${ }^{24}$ In a variety of cancers, such as lobular breast carcinoma, diffuse gastric carcinoma, endometrial and ovarian carcinoma, and hepatocellular carcinoma, reduced expression of E-cadherin due to genetic mutations, in combination with loss of heterozygosity at the E-cadherin gene (CDH1), has been correlated with the disruption of cell-cell contacts, epithelialmesenchymal transition, invasiveness, and metastatic potential. ${ }^{20,25-30}$ In this study, hepatocytes in normal liver and in hepatocellular adenoma showed a uniformly high expression of E-cadherin. Hepatocellular carcinoma with cirrhosis had a significantly 
Table 3 Value of selected markers in the diagnosis and differential diagnosis of primary hepatocellular neoplasms

\begin{tabular}{|c|c|c|c|c|c|c|}
\hline Markers & Value & $\begin{array}{l}\text { Hepatocellular } \\
\text { adenoma from } \\
\text { normal }\end{array}$ & $\begin{array}{l}\text { Hepatocellular } \\
\text { adenoma from } \\
\text { hepatocellular } \\
\text { carcinoma with } \\
\text { cirrhosis }\end{array}$ & $\begin{array}{l}\text { Hepatocellular } \\
\text { adenoma from } \\
\text { hepatocellular } \\
\text { carcinoma } \\
\text { without cirrhosis }\end{array}$ & $\begin{array}{l}\text { Hepatocellular } \\
\text { carcinoma with } \\
\text { cirrhosis from } \\
\text { normal }\end{array}$ & $\begin{array}{l}\text { Hepatocellular } \\
\text { carcinoma without } \\
\text { cirrhosis from } \\
\text { normal }\end{array}$ \\
\hline \multirow[t]{3}{*}{ E-cadherin } & SE\% (CI\%) & 70 (34-93) & 70 (35-93) & 70 (35-93) & $48(33-63)$ & $24(11-41)$ \\
\hline & SP\% (CI\%) & $55(32-77)$ & $77(62-89)$ & $56(38-73)$ & $85(62-97)$ & $85(62-97)$ \\
\hline & $\mathrm{ROC}(\mathrm{CI})$ & $0.63(0.44-0.81)$ & $0.74(0.57-0.9)$ & $0.63(0.46-0.8)$ & $0.66(0.55-0.77)$ & $0.54(0.44-0.65)$ \\
\hline \multirow[t]{3}{*}{ MMP-7 } & $\mathrm{SE} \%(\mathrm{CI} \%)$ & $100(69-100)$ & $100(69-100)$ & $100(69-100)$ & NA & NA \\
\hline & SP\% (CI\%) & $85(62-97)$ & $77(62-89)$ & $68(50-83)$ & & \\
\hline & ROC (CI) & $0.92(0.84-1.0)$ & $0.89(0.82-0.95)$ & $0.84(0.76-0.92)$ & & \\
\hline \multirow[t]{3}{*}{ MMP-9 } & SE\% (CI\%) & $80(44-98)$ & $80(44-97)$ & $80(44-98)$ & $68(52-81)$ & 85 (69-95) \\
\hline & SP\% (CI\%) & $25(0.9-49)$ & $68(52-81)$ & 85 (69-95) & 75 (51-91) & 75 (51-91) \\
\hline & $\mathrm{ROC}(\mathrm{CI})$ & $0.53(0.36-0.69)$ & $0.74(0.59-0.89)$ & $0.83(0.68-0.97)$ & $0.72(0.6-0.84)$ & $0.80(0.69-0.92)$ \\
\hline \multirow[t]{3}{*}{ MMP-7+E-cadherin } & SE\% (CI\%) & $70(35-93)$ & $70(35-93)$ & $70(35-93)$ & NA & NA \\
\hline & SP\% (CI\%) & $95(75-100)$ & $91(78-98)$ & $88(73-97)$ & & \\
\hline & $\mathrm{ROC}(\mathrm{CI})$ & $0.82(0.67-0.98)$ & $0.8(0.65-0.96)$ & $0.79(0.63-0.95)$ & & \\
\hline \multirow[t]{3}{*}{ MMP-9+E-cadherin } & $\mathrm{SE} \%(\mathrm{CI} \%)$ & $60(26-88)$ & $60(26-88)$ & $60(26-88)$ & $34(21-50)$ & $21(9-38)$ \\
\hline & SP\% (CI\%) & $65(41-85)$ & 96 (85-99) & $94(80-99)$ & $90(68-99)$ & $90(68-99)$ \\
\hline & ROC (CI) & $0.63(0.43-0.82)$ & $0.78(0.61-0.94)$ & $0.77(0.61-0.94)$ & $0.62(0.52-0.72)$ & $0.55(0.46-0.65)$ \\
\hline \multirow[t]{3}{*}{ MMP-7+MMP-9 } & SE\% (CI\%) & 80 (44-98) & 80 (44-98) & 80 (44-98) & NA & NA \\
\hline & SP\% (CI\%) & $100(83-100)$ & $100(92-100)$ & $100(90-100)$ & & \\
\hline & $\mathrm{ROC}(\mathrm{CI})$ & $0.9(0.77-1.0)$ & $0.9(0.77-1.0)$ & $0.9(0.77-1.0)$ & & \\
\hline \multirow{3}{*}{$\begin{array}{l}\text { MMP-7+MMP-9+ } \\
\text { E-cadherin }\end{array}$} & SE\% (CI\%) & $60(26-87)$ & $60(26-88)$ & $60(26-88)$ & NA & NA \\
\hline & SP\% (CI\%) & $100(83-100)$ & $100(92-100)$ & $100(90-100)$ & & \\
\hline & ROC (CI) & $0.8(0.64-0.96)$ & $0.8(0.64-0.96)$ & $0.8(0.64-0.96)$ & & \\
\hline
\end{tabular}

CI, confidence interval; NA, not applicable; ROC, receiver operating characteristics; SE, sensitivity; SP, specificity.

E-cadherin $=3$, MMP-7 $<1$ and MMP-9 $\geq 2$ were used as the diagnostic criteria for hepatocellular adenoma and for differentiating hepatocellular adenoma from normal, hepatocellular carcinoma with cirrhosis and hepatocellular carcinoma without cirrhosis.

E-cadherin $<2$ and MMP-9 $<2$ were used for differentiating both hepatocellular carcinoma with cirrhosis and hepatocellular carcinoma without cirrhosis from normal.

lower E-cadherin expression than normal liver and adenoma. The relatively higher level of E-cadherin expression in hepatocellular carcinoma without cirrhosis than hepatocellular carcinoma with cirrhosis, although statistically insignificant, could be partially responsible for the less aggressive behavior of hepatocellular carcinoma without cirrhosis.

MMPs are a group of zinc-dependent endopeptidases that share many structural and functional properties, but have different substrate specificities. ${ }^{15,31}$ They are historically divided on the basis of their specificity for extracellular matrix components into collagenases (MMP-1, -2, and -9), gelatinases (MMP-2, -3, and -9), stromelysins (MMP-3, -10, and -11), and matrilysin (MMP-7). ${ }^{32-34}$ As the list of MMP substrates grows (21 human MMPs have been identified thus far), they have been grouped into eight distinct structural classes; five are secreted and three are membrane type..$^{35}$ Enhanced MMP expression has been reported in various human malignant tumors. Studies of the association of MMPs with hepatocellular carcinoma have generally focused on the following three aspects: (1) those associated with carcinogenesis including overexpression of MMP-2 and MT1-MMP, (2) those associated with tumor progression including overexpression of MMP-2, -3, -9 , and MT1-MMP, and (3) those associated with invasion and metastasis including overexpression of MMP-2, -3 , and $-9 .{ }^{36-40}$ In this study, the expression of MMP-7 and MMP-9 showed a statistically significant difference between the different types of primary hepatocellular neoplasms.

MMP-7 is the smallest known MMP (28 kDa) and has a wide variety of substrates, including fibronectin, laminin, collagen type IV, gelatin, and proteoglycans. ${ }^{41}$ One unique aspect of MMP-7 is its exclusive expression in the tumor epithelial cells, whereas other MMPs may be present in both tumor epithelial and stromal cells. ${ }^{42,43}$ Therefore, a comparative analysis is more straightforward for MMP-7 than for other types of MMPs. MMP-7 has been reported to be involved in the invasion and metastasis of a variety of malignant neoplasms, including breast, colon, stomach, lung, skin, and prostate cancers. ${ }^{41}$ Increased MMP-7 expression in hepatocellular carcinoma and its association with the progression of hepatocellular carcinoma have been reported by us and by other investigators. ${ }^{20,40,44,45}$ In this study, the complete absence of MMP-7 in hepatocellular adenoma clearly separates 
it from normal liver, hepatocellular carcinoma with cirrhosis, and hepatocellular carcinoma without cirrhosis. If this observation can be verified in larger scale studies, MMP-7 could be a useful immunohistochemical marker in the diagnosis of hepatocellular adenoma and in differentiating hepatocellular adenoma from hepatocellular carcinoma.

MMP-9 is also known as gelatinase B or $92-\mathrm{kDa}$ type IV collagenase. Increased MMP-9 mRNA, tissue protein expression, and plasma levels have been shown to be associated with capsular invasion, portal vein invasion, and poor survival. . $^{36,39,46}$ MMP-9 gene expression can be induced by several stimulators including (1) the HBV virus X protein, which stimulates NF- $\kappa \mathrm{B}$ and AP-1 activation through the phosphatidylinositol 3-kinase (PI3K)/ Akt and ERKs pathway; (2) phorbol ester, which stimulates through protein kinase C-dependent activation of the Ras/ERK signaling pathway; (3) radiation, which stimulates through the PI3K/Akt/ $\mathrm{NF}-\kappa \mathrm{B}$ signal transduction pathway; (4) the TGF- $\beta$ activated p38 pathway; and (5) ethanol metabolite acetaldehyde, which increases NF- $\kappa \mathrm{B}$ and AP-1 activity through the $\mathrm{I} \kappa \mathrm{B}, \mathrm{JNK} / \beta$-TrCP, and p38 pathways. ${ }^{47-51}$ As these MMP-9 stimulators can also cause liver cirrhosis, it is conceivable that MMP-9 expression could be higher in hepatocellular carcinoma with cirrhosis than in hepatocellular carcinoma without cirrhosis. In this study, hepatocellular carcinoma without cirrhosis showed a significantly lower level of MMP-9 expression, which could be partially responsible for the less aggressive behavior seen in hepatocellular carcinoma without cirrhosis when compared with hepatocellular carcinoma with cirrhosis.

Using a standard formula, we analyzed the sensitivity, specificity, and ROCs of selected markers in the diagnosis and differential diagnosis of primary hepatocellular neoplasms. We have found that MMP-7 $<1$ alone is the most sensitive, whereas MMP-7 $<1+$ MMP-9 $\geq 2$ is the most specific marker distinguishing hepatocellular adenoma from normal, hepatocellular carcinoma with cirrhosis, and hepatocellular carcinoma without cirrhosis. MMP$9<2$ alone has the highest sensitivity especially for differentiating hepatocellular carcinoma without cirrhosis from normal. The combination of Ecadherin $<2$ and MMP- $9<2$ can differentiate both hepatocellular carcinoma with cirrhosis and hepatocellular carcinoma without cirrhosis from normal with high specificity, but the sensitivity was very low. The different clinical scenario and particular histological challenges in each individual case will determine the choice of markers ordered. For the purpose of detecting a tumor, markers with a higher sensitivity should be chosen. For the purpose of distinguishing one tumor type from the other, markers with a higher specificity should be used.

The major diagnostic challenge clinically is to differentiate between hepatocellular adenoma and a really well-differentiated hepatocellular carcinoma.
In our earlier study, we have shown a relatively higher expression of E-cadherin in low-grade hepatocellular carcinoma than in high-grade hepatocellular carcinoma. ${ }^{20}$ Therefore, we must exercise caution when using E-cadherin to differentiate well-differentiated hepatocellular carcinoma from adenoma. We have also noted a significantly higher MMP-7 expression in poorly differentiated hepatocellular carcinoma in comparison with well-differentiated hepatocellular carcinoma. ${ }^{20}$ However, as hepatocellular adenoma lacks MMP-7 expression, MMP-7 appears to be a more robust marker for this purpose. However, the true utility of these markers needs to be proven in further studies of small biopsy specimens and of histologically ambiguous cases.

In summary, we have studied the tissue expression of E-cadherin and MMPs in normal liver tissue, hepatocellular adenoma, hepatocellular carcinoma with cirrhosis, and hepatocellular carcinoma without cirrhosis. Hepatocellular adenoma was characterized by the complete absence of MMP-7 expression. Hepatocellular carcinoma with cirrhosis was characterized by a significantly lower E-cadherin expression and significantly higher MMP-7 expression when compared with hepatocellular adenoma. Hepatocellular carcinoma without cirrhosis was characterized by a significantly higher MMP-7 expression and a significantly lower MMP-9 expression when compared with hepatocellular adenoma. E-cadherin $=3, \mathrm{MMP}-7<1$, and MMP- $9 \geq 2$ can be used as the diagnostic criteria for hepatocellular adenoma and for distinguishing hepatocellular adenoma from normal, hepatocellular carcinoma with cirrhosis, and hepatocellular carcinoma without cirrhosis. E-cadherin $<2$ and MMP- $9<2$ can be used for distinguishing both hepatocellular carcinoma with cirrhosis and hepatocellular carcinoma without cirrhosis from normal. Although statistically not significant, hepatocellular carcinoma without cirrhosis showed a higher E-cadherin expression and a lower MMP-9 expression than hepatocellular carcinoma with cirrhosis, which could be partially responsible for the less aggressive behavior seen in hepatocellular carcinoma without cirrhosis when compared with hepatocellular carcinoma with cirrhosis.

\section{References}

1 Parkin DM, Bray F, Ferlay J, et al. Global Cancer Statistics, 2002. CA Cancer J Clin 2005;55:74-108.

2 Chen CJ, Wang LY, Lu SN, et al. Elevated aflatoxin exposure and increased risk of hepatocellular carcinoma. Hepatology 1996;24:38-42.

3 Harris CC. Hepatocellular carcinogenesis: recent advances and speculations. Cancer Cells 1990;2:146-148.

4 Johnson PJ, Williams R. Cirrhosis and the etiology of hepatocellular carcinoma. J Hepatol 1987;4:140-147.

5 Saito I, Miyanmura T, Ohabayashi A, et al. Hepatitis C virus infection is associated with the development of 
hepatocellular carcinoma. Proc Natl Acad Sci USA 1990;87:6547-6549.

6 Nazeako UC, Goodman ZD, Ishak KG. Hepatocellular carcinoma in cirrhotic and noncirrhotic livers. A clinicohistopathologic study of 804 North American patients. Am J Clin Pathol 1996;105:65-75.

7 Evert M, Dombrowski F. Hepatocellular carcinoma in the non-cirrhotic liver. Pathologe 2008;29:47-52.

8 Lee PJ. Glycogen storage disease type I: pathophysiology of liver adenomas. Eur J Pediatr 2002;161(Suppl 1): S46-S49.

9 Edmonston H, Henderson B, Benton B. Liver-cell adenomas associated with use of oral contraception. N Engl J Med 1976;294:470-472.

$10 \mathrm{Kew}$ MC. Hepatocellular carcinoma with and without cirrhosis. A comparison in southern African blacks. Gastroenterology 1989;97:136-139.

11 Okuda K, Nakashima T, Koijiro M, et al. Hepatocellular carcinoma without cirrhosis in Japanese patients. Gastroenterology 1989;97:140-146.

$12 \mathrm{Gao} Z \mathrm{ZH}$. Predictive markers for the recurrence of hepatocellular carcinoma. J Surg Oncol 2005;92: 274-275.

13 Kakar S, Burgart LJ, Batts KP, et al. Clinicopathologic features and survival in fibrolamellar carcinoma: comparison with conventional hepatocellular carcinoma with and without cirrhosis. Mod Pathol 2005;18:1417-1423.

14 Ferrel LD. Hepatocellular carcinoma arising in a focus of multilobular adenoma: a case report. Am J Surg Pathol 1993;17:525-529.

15 Brikedal-hansen H, Moore WGI, Bodden MK, et al. Matrix metalloproteinases: a review. Crit Rev Oral Biol Med 1993;4:197-250.

16 Docherty AJP, O’Conell J, Crabbe T, et al. The matrix metalloproteinases and their natural inhibitors: prospects for treating degenerative tissue diseases. Trends Biotechnol 1992;10:200-207.

17 Senta $\mathrm{A}$, Itoh $\mathrm{F}$, Yamamoto $\mathrm{H}$, et al. Relation of matrilysin messenger RNA expression with invasive activity in human gastric cancer. Clin Exp Metastasis 1998;16:313-321.

18 Yoshimoto M, Itoh F, Yamamoto H, et al. Expression of MMP-7 (Pump-1) mRNA in human colorectal cancers. Int J Cancer 1993;54:614-618.

19 Wijnhoven BPL, Dinjens WNM, Pignatelli M. Ecadherin-cateinin cell-cell adhesion complex and human cancer. Br J Surg 2000;87:992-1005.

20 Gao ZH, Trekiakova M, Liu WH, et al. Association of Ecadherin, matrix metalloproteinases and tissue inhibitors of metalloproteinases with the progression and metastasis of hepatocellular carcinoma. Mod Pathol 2006;19:533-540.

21 Hirohashi S, Ishak KG, Kojiro M, et al. Hepatocellular carcinoma In: Hamilton SR and Aaltonen LA (eds). World Health Organization Classification of Tumors. Pathology and Genetics of Tumors of the Digestive System. IARC Press: Lyon, 2000;165-166.

22 Altman DG, Bland JM. Diagnostic tests. 1: Sensitivity and specificity. BMJ 1994;308:1552.

23 Zweig MH, Campbell G. Receiver-operating characteristic (ROC) plots: a fundamental evaluation tool in clinical medicine. Clin Chem 1993;39: 561-577.

24 Kemler R. From cadherins to catenins: cytoplasmic protein interactions and regulation of cell adhesion. Trends Genet 1993;9:317-321.
25 Berx G, Becker KF, Hofler H, et al. Mutations of human E-cadherin (CDH1) gene. Hum Mutat 1998;12:226-237.

26 Berx G, Cleton-Jansen AM, Noller F, et al. E-cadherin is a tumor/invasion suppressor gene mutated in human lobular breast cancers. EMBO J 1995;14:6107-6115.

27 Inayoshi J, Ichida T, Sugitani S, et al. Gross appearance of hepatocellular carcinoma reflects E-cadherin expression and risk of early recurrence after surgical treatment. J Gastroenterol Hepatol 2003;18:673-677.

28 Matsumura T, Makino R, Mitamura K. Frequent downregulation of E-cadherin by genetic and epigenetic changes in malignant progression of hepatocellular carcinomas. Clin Cancer Res 2001;7:594-599.

29 Saeki Y, Hazeki K, Matsumoto M, et al. Correlation between metastatic potency and the down-regulation of E-cadherin in the mouse hepatoma cell lines G-1 and G-5. Oncol Rep 2000;7:731-735.

30 Shinoyama Y, Hirohashi S. Cadherin intercellular adhesion molecule in hepatocellular carcinomas: loss of E-cadherin in an indifferentiated carcinoma. Cancer Lett 1991;57:131-135.

31 Lichtinghagen R, Helmbrecht $\mathrm{T}$, Arndt $\mathrm{B}$, et al. Expression pattern of matrix metalloproteinases in human liver. Eur J Clin Chem Clin Biochem 1995;33:65-71.

32 Cottam DW, Rees RC. Regulation of matrix metalloproteinases: their role in tumor invasion and metastasis. Int J Oncol 1993;2:861-872.

33 Curran S, Murray GI. Matrix metalloproteinases: molecular aspects of their roles in tumor invasion and metastasis. Eur J Cancer 2000;36:1621-1630.

34 Egeblad M, Werb Z. New functions for the matrix metalloproteinases in cancer progression. Nat Rev Cancer 2002;2:161-174.

35 Yang W, Arii S, Gorrin-Rivas MJ, et al. Human macrophage metalloelastase gene expression in colorectal carcinoma and its clinicopathologic significance. Cancer 2001;91:1277-1283.

36 Arri S, Mise M, Harada T, et al. Overexpression of matrix metalloproteinase 9 gene in hepatocellular carcinoma with invasive potential. Hepatology 1996;24:316-322.

37 Giannelli G, Bergamini C, Marinosci F, et al. Clinical role of MMP-2/TIMP-2 imbalance in hepatocellular carcinoma. Int J Cancer 2002;97:425-431.

38 Grigioni WF, Garisa S, D'Errico A, et al. Evaluation of hepatocellular carcinoma aggressiveness by a panel of extracellular matrix antigens. Am J Pathol 1991;138:647-654.

39 Sakamoto Y, Mafune K, Mori M, et al. Overexpression of MMP-9 correlates with growth of small hepatocellular carcinoma. Int J Oncol 2000;17:237-243.

40 Yamamoto $\mathrm{H}$, Itoh $\mathrm{F}$, Adachi $\mathrm{Y}$, et al. Relation of enhanced secretion of active matrix metalloproteinases with tumor spread in human hepatocellular carcinoma. Gastroenterology 1997;112:1290-1296.

41 Wilson CL, Matrisian LM. Matrilysin: an epithelial matrix metalloproteinase with potentially novel functions. Int J Biochem Cell Biol 1996;28:123-136.

42 Saariaho-Kere UK, Crouch EC, Parks WC. Matrix metalloproteinase matrilysin is constitutively expressed in adult human exocrine epithelium. J Invest Dermatol 1995;105:190-196.

43 Wilson CL, Hepper KJ, Rudolph LA, et al. The metalloproteinase matrilysin is preferentially expressed by epithelial cells in a tissue-restricted pattern in the mouse. Mol Biol cell 1995;6:851-869. 
44 Ishii Y, Nakasato Y, Kobayashi S, et al. A study on angiogenesis-related matrix metalloproteinase networks in primary hepatocellular carcinoma. J Exp Clin Cancer Res 2003;22:461-470.

45 Ozaki I, Mizuta T, Zhao G, et al. Involvement of the Ets-1 gene in overexpression of matrilysin in human hepatocellular carcinoma. Cancer Res 2000;60: 6519-6525.

46 Hayasaka A, Suzuki N, Fujimoto N, et al. Elevated plasma levels of matrix metalloproteinase-9 (92-kd type IV collagenas B ) in hepatocellular carcinoma. Hepatology 1996;24:1058-1062.

47 Cheng JC, Chou CH, Kuo ML, et al. Radiationenhanced hepatocellular carcinoma cell invasion with MMP-9 expression through PI3K/Akt/NF- $\kappa \mathrm{B}$ signal transduction pathway. Oncogene 2006;25:7009-7018.

48 Chung TW, Lee YC, Kim CH. Hepatitis B viral HBX induces matrix metalloproteinase-9 gene expression through activation of ERK and PI-3K/AKT pathways: involvement of invasive potential. FASEB J 2004;18: 1123-1125.

49 Hsiang CY, Wu SL, Chen JC, et al. Acetaldehyde induces matrix metalloproteinase-9 gene expression via nuclear factor- $\kappa \mathrm{B}$ and activator protein 1 signaling pathway in human hepatocellular carcinoma cells: association with invasive potential. Toxicol Lett 2007;171:78-86.

50 Kim ES, Kim MS, Moon A. TGF- $\beta$-induced upregulation of MMP-2 and MMP-9 depends on p38 MAPK, but not ERK signaling in MCF10A human breast epithelial cells. Int J Oncol 2004;25:1375-1382.

51 Liu S, Crepin M, Liu JM, et al. FGF-2 and TPA induce matrix metalloproteinase-9 secretion in MCF-7 cells through PKC activation of the Ras/ERK pathway. Biochem Biophys Res Commun 2002;293: 1174-1182. 